\title{
The Equivalence of Causality Detection in VAR and VECM Modeling with Applications to Exchange Rates
}

\author{
T.J. Brailsford \\ UQ Business School, University of Queensland, Australia \\ J. H.W. Penm \\ The Australian National University, Australia \\ R.D. Terrell \\ The Australian National University, Australia
}

\begin{abstract}
Vector error-correction models (VECM) are increasingly being used to capture dynamic relationships between financial variables. Estimation and interpretation of such models can be enhanced if zero restrictions are allowed in the coefficient matrices. Specifically, in tests of indirect causality and/or Granger non-causality in a VECM, the efficiency of the causality detection is crucially dependent upon finding zero coefficient entries where the true structure does indeed include zero entries. Such a VECM is referred to as a zero-non-zero (ZNZ) patterned VECM and includes full-order models. Recent advances have shown how ZNZ patterns can be explicitly recognized in a VECM and used to provide an effective means of detecting Granger-causality, Granger non-causality and indirect causality. This paper develops a general approach and framework for $I(d)$ integrated systems. We show that causality detection in an $I(d)$ system can be discovered identically from the ZNZ patterned VECM's or the equivalent VAR models (JEL: C10, C63, F30, G10).
\end{abstract}

Keywords: error correction models, VAR, granger causality, purchasing power parity.

\section{Introduction}

The use of vector autoregressive models (VAR) and vector errorcorrection models (VECM) for analyzing dynamic relationships among

(Multinational Finance Journal, 2006, vol.10, no. 3/4, pp. 153-177)

(C) Multinational Finance Society, a nonprofit corporation. All rights reserved. DOI: $10.17578 / 10-3 / 4-1$ 
financial variables has become common in the literature (e.g. MacDonald and Power [1995], Lee [1996], Barnhill et al. [2000]). Moreover, relationships are often examined within the framework of a cointegrated system. The popularity of these models has been associated with the realization that financial systems and relationships among financial variables are complex, which traditional time-series models have failed to fully capture.

Engle and Granger (1987) note that, for cointegrated systems, the VAR in first differences will be mis-specified and the VAR in levels will ignore important constraints on the coefficient matrices. Although these constraints may be satisfied asymptotically, efficiency gains and improvements in forecasts are likely to result from their imposition. Hence, Engle and Granger (1987) suggest that if a time-series system under study includes integrated variables of order 1 and cointegrating relations, then this system will be more appropriately specified as a vector error-correction model (VECM) rather than a VAR. Comparisons of forecasting performance of VECMs versus VARs for cointegrated systems are reported in Engle and Yoo (1987), and LeSage (1990). ${ }^{1}$ The results of these studies indicate that the VECM is a more appropriate specification in terms of smaller long-term forecast errors, when the variables satisfy cointegration conditions.

Subsequently, Ahn and Reinsel (1990), and Johansen (1988, 1991) have proposed various algorithms for the estimation of cointegrating vectors in full-order VECM models, which contain all non-zero entries in the coefficient matrices. There are many examples of the use of fullorder VECM models in the analysis of short-term dynamics and longterm cointegrating relationships (e.g. Reinsel and Ahn [1992]; Johansen [1992, 1995]).

A problem can arise in relation to the use of full-order VECM models as such models assume nonzero elements in all their coefficient matrices. As the number of elements to be estimated in these possibly over-parameterised models grows with the square of the number of variables, the degrees of freedom is heavily reduced.

A related problem is to provide satisfactory financial and economic interpretations for the estimated cointegrating vectors. As emphasized by Penm et al. (1997) it is important to introduce a priori information, usually to produce zero-non-zero (ZNZ) patterns. To address this issue,

1. Christoffersen and Diebold (1998) indicate that imposing cointegration on a bivariate system can improve forecasts. 
Penm et al. present a search algorithm and procedure to identify the optimal specification of a ZNZ patterned VECM for an $I(1)$ system. $^{2}$

Application of VECM models to economic and financial time-series data have revealed that zero coefficient entries are indeed possible (Chow [1983], King et al. [1991]). ${ }^{3}$ An optimal VECM specification with zero entries suggests that the cointegrating vectors and the loading vectors may also contain zero entries. In this approach the zero entries are determined from data, with model selection criteria used for the selection of the optimal model (Penm et al. [1997]). However, the existence of zero entries has not been fully discussed in causality and cointegration theory. Specifically the ability to detect the presence or absence of indirect causality and/or Granger non-causality is related to the identification of the optimal model. Further, the exact nature of the long-term cointegration relations will be crucially dependent upon finding those zero coefficient entries where the true structure does indeed include such zero entries.

The paper's main contribution is to demonstrate that Granger noncausality, indirect causality and the ZNZ patterned cointegrating vectors can be detected in the context of a single ZNZ patterned VECM framework which allows for zero entries, that is, for time series of integrated order $I(d), d \geq 1$. Moreover, the Granger causal relations are detected from the coefficient matrices on the lagged difference terms and from the error-correction terms. The paper also shows that identical causality detection for this $I(d)$ system can be revealed in the equivalent VAR framework.

The remainder of this paper is organized as follows. Section II reviews causality patterns in VAR modeling. Section III describes zero entries in a ZNZ patterned VAR and its equivalent VECM for an $\mathrm{I}(d)$ system. Causality detection in VECM modeling is also discussed. ${ }^{4}$ Two three-asset examples are then presented for illustrative purposes. To

2. Of note, an $I(1)$ system does not contain any fractionally integrated variables.

3. Chow (1983) examines Granger causality in a bi-variate time-series model, and discusses the use of zero restrictions to represent causality interaction in different but equivalent model specifications. Chow's models are not set up to separate the dynamic and long-term responses. However, VECM models proposed in this paper accommodate both long-term and dynamic responses.

4. There are two appendices to this paper which are available upon request. Appendix A outlines the algorithm proposed by Penm et al. (1997) for the selection of the optimal VECM. Appendix B describes the use of the Yule-Walker relations for fitting ZNZ patterned VECM models. 
demonstrate the usefulness of the ZNZ patterned VECM for causality detection and cointegration investigation, section IV demonstrates two exchange rate applications. The first application examines the causal relationships between the movements of the Euro's exchange rate and the money supply. The second application conducts an examination of purchasing power parity focusing on the Yen. Concluding remarks are provided in section $\mathrm{V}$.

\section{Causality Patterns in VAR Modeling}

First, as shown below, note that a VECM is identical to a VAR model with unit roots. Consider the following VAR model:

$$
y(t)+\sum_{\tau-1}^{p} A_{\tau} y(t-\tau)=A^{p}(L) y(t)=\varepsilon(t)
$$

where $\mathcal{E}(t)$ is a $(s x 1)$ independently and identically distributed vector random process with $E\{\boldsymbol{\varepsilon}(t)\}=0$ and $E\left\{\boldsymbol{\varepsilon}(t) \mathcal{E}^{\prime}(t-\tau)\right\}=V, \tau=0$, and $E\left\{\boldsymbol{\varepsilon}(t) \mathcal{E}^{\prime}(t-\tau)\right\}=0, \tau>0$.

$A_{\tau}, \tau=1,2, \ldots p$ are (sxs) parameter matrices, and, $A^{p}(L)=I+\sum_{\tau-1}^{p} A_{\tau} L^{\tau}$

$L$ denotes the lag operator and the roots of $\left|A^{P}(L)\right|=0$ lie outside or on the unit circle. Further, we have the following relation:

$$
A^{p}(L)=A^{p}(1)+(I-L)\left(I+\sum_{\tau-1}^{p-1} A_{\tau}^{*} L^{\tau}\right)
$$

It follows from the concept of cointegrated variables that $y(t)$ is said to be $I(1)$ if it contains at least one element which must be differenced before it becomes $I(0)$ (Granger [1981]). Then $y(t)$ is said to be cointegrated of order 1 with the cointegrating vector, $\beta$, if $\beta^{\prime} y(t)$ becomes $I(0)$, where $y(t)$ has to contain at least two $I(1)$ variables. Under this assumption the identical VECM for (1) can be described as:

$$
A^{*} y(t-1)+A^{p-1}(L) \Delta y(t)=\varepsilon(t)
$$

where $y(t)$ contains both $I(0)$ and $I(1)$ variables, $\Delta=(I-L), A^{*}=A^{p}(1)$, 
$A^{*} y(t-1)$ is stationary, and,

$$
A^{p-1}(L)=I+\sum_{\tau-1}^{p-1} A_{\tau}^{*} L^{\tau}
$$

The first term in (2) (i.e. $\left.A^{*} y(t-1)\right)$ is the error-correction term, which contains the long-term cointegrating relationships. $A^{p-1}(L) \Delta y(t)$ is referred to as the VAR part of the VECM, describing the short-term dynamics. Because $y(t)$ is cointegrated of order 1, the long-term impact matrix $A^{*}$ must be singular. As a result $A^{*}=\alpha \beta^{\prime}$, where $\alpha$ and $\beta$ are (sxr) matrices and the rank of $A^{*}$ is $r$, where $r<s$. The columns of $\beta$ are the cointegrating vectors, and the rows of $\alpha$ are the loading vectors.

Now, consider a bi-variate system where $y(t)=\left[y_{1}(t) y_{2}(t)\right]^{\prime}$, then the following natural way of defining a causal ordering may be developed.

Consider $\mathrm{a}_{i j}^{p}(L)=\sum_{\tau=1}^{p} \alpha_{i j}^{\tau} L^{\tau}$, where $\mathrm{a}_{i j}^{p}(L)$ is the $(i, j)$-th entry of $A^{p}(L)$.

Definition (a): $y_{1}(t)$ Granger non-causes $y_{2}(t)$, and $y_{2}(t)$ Granger causes $y_{1}(t)$ if and only if $a_{21}^{p}(L)=0$ and at least one $\alpha_{12}^{\tau}, \tau=1, \ldots, p$, is nonzero. That means: $A^{p}(L)=\left[\begin{array}{cc}\mathrm{a}_{11}^{p}(L) & \mathrm{a}_{12}^{p}(L) \\ 0 & \mathrm{a}_{22}^{p}(L)\end{array}\right]$ and the coefficients, $\alpha_{12}^{\tau}, \tau=1, \ldots, p$ in $\alpha_{12}^{p}(L)$ can be either zero or nonzero, but at least one $\alpha_{12}^{\tau}$ is nonzero. Further, there exist $2^{p-1}$ different patterns of $\mathrm{a}_{1,2}^{p}(L)$ in this bi-variate system, indicating that $y_{1}(t)$ Granger non-causes $y_{2}(t)$, and $y_{2}(t)$ Granger causes $y_{1}(t)$.

Definition (b): $y_{2}(t)$ Granger non-causes $y_{1}(t)$, and $y_{1}(t)$ Granger causes $y_{2}(t)$ if and only if $\mathrm{a}_{12}^{p}(L)=0$ and at least one $\alpha_{21}^{\tau}, \tau=1, \ldots, p$, is nonzero.

That means: $A^{p}(L)=\left[\begin{array}{cc}\mathrm{a}_{11}^{p}(L) & 0 \\ \mathrm{a}_{21}^{p}(L) & \mathrm{a}_{22}^{p}(L)\end{array}\right]$

and the coefficients, $\alpha_{21}^{\tau}, \tau=1, \ldots, p$ in $\mathrm{a}_{21}^{p}(L)$ can be either zero or nonzero, but at least one $\alpha_{21}^{\tau}$ is nonzero.

Definition (c): $y_{2}(t)$ Granger causes $y_{1}(t)$ and $y_{1}(t)$ Granger causes $y_{2}(t)$ if and only if $a_{12}^{p}(L) \neq 0$ and $a_{21}^{p}(L) \neq 0$.

Definition (d): $y_{2}(t)$ Granger non-causes $y_{1}(t)$ and $y_{1}(t)$ Granger noncauses $y_{2}(t)$ if and only if $a_{12}^{p}(L)=0$ and $a_{21}^{p}(L)=0$.

The above causality patterns can be detected from the optimal selected ZNZ patterned VAR proposed in Penm and Terrell (1984b). More general causal patterns can be treated using definitions suggested 
by Hsiao (1982).

Consider the following trivariate system:

$$
\left[\begin{array}{ccc}
a_{11}^{p}(L) & a_{12}^{p}(L) & a_{13}^{p}(L) \\
a_{21}^{p}(L) & a_{22}^{p}(L) & a_{23}^{p}(L) \\
0 & a_{32}^{p}(L) & a_{33}^{p}(L)
\end{array}\right]\left[\begin{array}{l}
y_{1}(t) \\
y_{2}(t) \\
y_{3}(t)
\end{array}\right]=\varepsilon(t),
$$

which describes $y_{1}(t)$ causing $y_{3}(t)$ but only through $y_{2}(t)$. In this trivariate system the above indirect causality implies:

$$
\mathrm{a}_{31}^{p}(L)=0, \mathrm{a}_{21}^{p}(L) \neq 0 \text { and } \mathrm{a}_{32}^{p}(L) \neq 0 \text {. }
$$

Also

$\left\{\alpha_{12}^{\tau}=0\right.$ or $\left.\neq 0\right\},\left\{\alpha_{13}^{\tau}=0\right.$ or $\left.\neq 0\right\}$ and $\left\{\alpha_{23}^{\tau}=0\right.$ or $\left.\neq 0\right\}, \tau=1, \ldots, p$.

The greater the number of components, $y_{i}(t), i=1,2, \ldots$, the more complicated are the causal patterns that may be detected. ${ }^{5}$

\section{Zero Entries in ZNZ Patterned VAR and Equivalent VECM for an $I(d)$ System}

This section of the paper consists of two parts. The first part shows the equivalence between ZNZ patterned VECMs and the corresponding VAR models. The second part presents two three-asset examples to demonstrate the equivalence. Thus this section contributes both theoretical development and numerical examples to show the equivalence of causality detection in VAR and VECM modeling.

\section{A. Equivalence Between VECM and VAR Models}

In an $I(d)$ system the equivalent VECM derived from (1) can be described as follows:

$$
\begin{aligned}
& A^{p}(1) y(t-1)+A^{p-1}(1) \Delta y(t-1)+\ldots \\
& +A^{p-d+1}(1) \Delta^{d-1} y(t-1)+A^{p-d}(L) \Delta^{d} y(t)=\varepsilon(t),
\end{aligned}
$$

$A^{p-i}(1) \Delta^{i} y(t-1)$ are stationary, $i=0, \ldots, d-1$. The first $d$ terms are the

5. For more detail, again refer to Penm and Terrell (1984b) and Hsiao (1982). 
error-correction terms, while $A^{p-d}(L) \Delta^{d} y(t)$ is said to be the autoregressive part of the model.

In order to show the equivalence between $\mathrm{ZNZ}$ patterned VECMs and the corresponding VAR models, the following two properties need to be established:

(i) If $y_{j}$ does not Granger-cause $y_{i}$ then every $(i, j)$-th entry must be zero for all coefficient matrices in the VAR. Also all $(i, j)$-th coefficient elements in the equivalent VECM are zeros.

(ii) If $y_{j}$ does Granger-cause $y_{i}$, then the $(i, j)$-th element of $A^{p}(L)$ in the VAR is nonzero. In addition at least a single $(i, j)$-th coefficient element is nonzero in $A_{p}(1), A^{p-1}(1), \ldots, A^{p-d+1}(1)$, or $A^{p-d}(L)$ in the equivalent VECM.

To prove Property $(i)$, we have the following relations in the VAR and its equivalent VECM:

$$
A^{k}(L)=A^{k}(1) L+A^{k-1}(L)(I-L), k=p, p-1, \ldots, p-d+1 .
$$

Since Granger causality detection is crucially dependent on the positions of off-diagonal zero entries in the coefficient matrices, we therefore focus on the positions where $i \neq j$. If the $(i, j)$-th entries of $A^{k}(L)$, $A^{k}(1)$, and $A^{k-1}(L)$ are $a_{i, j}(L), a_{i, j}(1)$, and $e_{i, j}(L)$ respectively, we have:

$$
a_{i, j}(L)=a_{i, j}(1) L+e_{i, j}(L)(1-L), i \neq j .
$$

Now we define $e_{i, j}(L)$ by:

and thus,

$$
e_{i, j}(L)=e_{1} L+\ldots+e_{k-1} L^{k-1}
$$

$$
e_{i, j}(L)(1-L)=e_{1} L+\left(e_{2}-e_{1}\right) L^{2}+\ldots+\left(e_{k-1}-e_{k-2}\right) L^{k-1}-e_{k-1} L^{k}
$$

If $a_{i j}(L)=0$, then $a_{i j}(1)$ will also be zero. From (5) we have $e_{i j}(L)(1-L)$ $=0$, and (6) produces $e_{1}=0, e_{2}-e_{1}=0, \ldots, e_{k-1}-e_{k-2}=0, e_{k-1}=0$, which lead to $e_{i}=0, i=1, \ldots, k-1$, and therefore $c_{i j}(L)=0$.

At this point, if the $(i, j)$-th entry of $A^{k}(L)$ is zero, then the $(i, j)$-th elements of both $A^{k}(1)$ and $A^{k-1}(L)$ are zeros. Therefore we can conclude that if every $(i, j)$-th entry is zero for all coefficient matrices in a VAR then all $(i, j)$-th coefficient elements in the error-correction terms and in the vector autoregressive part of the VECM, will also be zeros.

Analogously it is evident that if the $(i, j)$-th elements of all $A^{k}(1), k=$ 
$p, p-1, \ldots, p-d+1$ and $A^{k-1}(L)$ in (3) are zeros then the $(i, j)$-th entry of $A^{p}(L)$ in the equivalent VAR will be zero. Therefore we can conclude that if all $(i, j)$-th coefficient elements in the error-correction terms and all $(i, j)$-th coefficient elements in the vector autoregressive part of the VECM are zeros, then every $(i, j)$-th entry is zero for all coefficient matrices in a VAR. Thus we establish Property $(i)$.

To prove Property (ii), we can express (4) as follows:

$$
\begin{gathered}
A^{p}(L)=A^{p}(1) L+A^{p-1}(L)-A^{p-1}(L) L \\
A^{p-1}(L)=A^{p-1}(1) L+A^{p-2}(L)-A^{p-2}(L) L \\
\vdots \\
A^{p-d+1}(L)=A^{p-d+1}(1) L+A^{p-d}(L) L
\end{gathered}
$$

From (7.3) it is obvious that if the $(i, j)$-th element of $A^{p-d+1}(1)$ is nonzero, then the $(i, j)$-th element of $A^{p-d+1}(L)$ is nonzero. Also if the $(i, j)$-th element of $A^{p-d}(L)$ is nonzero, then a zero $(i, j)$-th element of $A^{p-d+1}(1)$ leads to a nonzero $(i, j)$ element of $A^{p-d+1}(L)$. Thus, we have proved that if there exists a nonzero $(i, j)$-th element in either $A^{k}(1)$ or $A^{k-1}(L), k=p, p-1, \ldots, p-d+1$ in (4), then the corresponding $(i, j)$-th element of $A^{k}(L)$ is nonzero. This outcome shows that if any single $(i, j)$ th element is nonzero in any one of the $d$ matrices, $A^{k}(1), k=p, p-$ $1, \ldots, p-d+1$, or $A^{p-d}(L)$ in the VECM in (3) is nonzero, then the $(i, j)$ th element of $A^{p}(L)$ in the equivalent VAR is nonzero.

Analogously from (7.1) if the $(i, j)$-th element of $A^{p}(L)$ is nonzero, then at least the $(i, j)$-th element is nonzero in one of the following $d$ coefficient matrices, or $A^{p-d}(L)$ :

$$
A^{p}(1), A^{p-1}(1), \ldots, A^{p-d+1}(1) .
$$

Therefore we have demonstrated that Property (ii) is established.

An indirect causality from $y_{j}$ to $y_{i}$ through $y_{m}$ indicates $y_{j}$ causing $y_{i}$ but only through $y_{m}$. Hence, $y_{j}$ Granger-causes $y_{m}, y_{m}$ Granger-causes $y_{i}$, and $y_{j}$ does not Granger-cause $y_{i}$ directly. It can be easily demonstrated that the VAR in (1) has nonzero $(m, j)$-th and $(i, m)$-th elements and a zero $(i, j)$-th element of $A^{p}(L)$. The identical indirect causality can also be shown in the equivalent VECM. Thus any questions on the nature of the causal pattern can be addressed either through the VAR approach or the VECM approach, and the causal pattern identified is identical. 
It is noteworthy that Johansen (1988) has proposed the following VECM equivalent to the VAR model of (1) in an I(1) system:

$$
\Gamma^{p-1}(L) \Delta y(t)+A^{*} y(t-p)=\varepsilon(t)
$$

where

$$
\Gamma^{p-1}(L)=I+\sum_{i=1}^{p-1} \Gamma_{i} L^{i}
$$

The error-correction term of this VECM is $A^{*} y(t-p)$, while the errorcorrection term in (2) is $A^{*} y(t-1)$. Thus we have:

$$
\Gamma_{k}=I_{p}+A_{1}+\cdots+A_{k}, k=1, \ldots, p-1,
$$

and

$$
A^{*}=I_{p}+A_{1}+\cdots+A_{p}
$$

Recall that $a_{i j}^{k}$ denotes the $(i, j)$-th entry of $A_{k}$. Let $g_{i j}^{k}$ and $a_{i j}^{*}$ denote the $(i, j)$-th entry of $\Gamma_{k}$ and $A^{*}$ respectively. From (9) it is obvious that all $p$ entries, $\left\{a_{i j}^{k}, k=1, \ldots, p-1\right\}$ are zeros; then $a_{i j}^{*}$ is zero and all $\left\{g_{i j}^{k}, k=\right.$ $1, \ldots, p\}$ are also zeros. Similarly if $a_{i j}^{*}$ and all $\left\{g_{i j}^{k}, k=1, \ldots, p-1\right\}$ are zeros, then $\left\{a_{i j}^{k}, k=1, \ldots, p\right\}$ are zeros. Therefore we can conclude that Property (i) is valid for (8) and the equivalent VAR. We then show that if any single $a_{i j}^{k}, k=1, \ldots, p$ in the VAR is nonzero, then the $(i, j)$-th entry is nonzero in either $A^{*}$ or $\Gamma^{p-1}(L)$. To begin with, we rewrite (9) as:

$$
\begin{gathered}
\Gamma_{1}=I_{p}+A_{1}, \\
\Gamma_{2}=\Gamma_{1}+A_{2}, \\
\ldots \\
\Gamma_{k}=\Gamma_{k-1}+A_{k}, k=2, \ldots, p-1
\end{gathered}
$$

From (11) if $a_{i j}^{1}$ is nonzero, then $g_{i j}^{1}$ is nonzero. However if $a_{i j}^{1}$ is zero, then $g_{i j}^{1}$ will be zero. We then inspect $a_{i j}^{2}$. Similarly, if $a_{i j}^{2}$ is nonzero, then $g_{i j}^{2}$ is nonzero. In addition, it is obvious that if $a_{i j}^{p}$ is nonzero and all $a_{i j}^{k}, k=1, \ldots, p-1$ are zeros, then $a_{i j}^{*}$ is nonzero, even though all $g_{i j}^{k}, k=$ $1, \ldots, p-1$ are zeroes.

Analogously it can be proved that if any single $(i, j)$-entry is nonzero in either $A^{*}$ or $\Gamma^{p-1}(L)$ then the $(i, j)$-th entry of $A^{p}(L)$ in the equivalent VAR is nonzero. Therefore, the above demonstrates that if $y_{j}$ does 
Granger-cause $y_{i}$ in $(8)$, then the $(i, j)$-th element of $A^{p}(L)$ in the VAR is nonzero. In addition the $(i, j)$-entry is also nonzero in either $A^{*}$ or $\Gamma^{p-1}(L)$ or both in the equivalent VECM. Thus the causal pattern identified through the VAR approach or the VECM approach is identical.

\section{B. Illustrations}

To show the equivalence of causality detection in VAR and VECM modeling, two three-asset examples are presented to illustrate this outcome. The first example involves two cointegrating relations and one unit root, while the second one involves one cointegrating relation and two unit roots.

In considering a VAR model with $\mathrm{ZNZ}$ patterned coefficient matrices, we allow for zero entries in the parameter matrices $A_{\tau}$ of (1). If $y_{1, t}, y_{2, t}$ and $y_{3, t}$ are the log prices of three assets, then the returns on the assets are defined by $\Delta y_{1, t}=z_{1, t}, \Delta y_{2, t}=z_{2, t}$, and $\Delta y_{3, t}=z_{3, t}$. All $z_{1, t}, z_{2, t}$ and $z_{3, t}$ are jointly determined by the following equations:

$$
\begin{gathered}
z_{1, t}-0.6 z_{1, t-1}-0.8 z_{2, t-1}=\varepsilon_{1, t} \\
z_{2, t}-0.1 z_{1, t-1}-0.4 z_{2, t-1}-0.8 z_{3, t-1}=\varepsilon_{2, t} \\
z_{3, t}-0.1 z_{2, t-1}-0.8 z_{3, t-1}=\varepsilon_{3, t}
\end{gathered}
$$

The equivalent VAR model of this system can then be presented as:

$$
\left[\begin{array}{c}
z_{1, t} \\
z_{2, t} \\
z_{3, t}
\end{array}\right]+\left[\begin{array}{ccc}
-0.6 & -0.8 & 0 \\
-0.1 & -0.4 & -0.8 \\
0 & -0.1 & -0.8
\end{array}\right]\left[\begin{array}{c}
z_{1, t-1} \\
z_{2, t-1} \\
z_{3, t-1}
\end{array}\right]=\left[\begin{array}{c}
\varepsilon_{1, t} \\
\varepsilon_{2, t} \\
\varepsilon_{3, t}
\end{array}\right] \text {. }
$$

In this VAR model, both $a_{1,3}^{1}(L)$ and $a_{3,1}^{1}(L)$ are zeros. Thus Granger noncausality exists between the first asset's return and the third asset's return. Further, $a_{2,1}^{1}(L) \neq 0$ and $a_{3,2}^{1}(L) \neq 0$, which indicate indirect causality from the first to the third asset's return via the second asset's return.

To inspect unit roots, we have the following relationship to determine the roots of the characteristic polynomial: 


$$
\operatorname{det}\left[\begin{array}{ccc}
1-0.6 L & -0.8 L & 0 \\
-0.1 L & 1-0.4 L & -0.8 L \\
0 & -0.1 L & 1-0.8 L
\end{array}\right]=0
$$

This relationship leads to $\operatorname{det}\left\{\left(1-0.8 L+0.08 L^{2}\right)(1-L)\right\}=0$. Therefore a single unit root is detected.

Next, we turn to the VECM modeling. By adding and subtracting a $\left[z_{1, t-1} z_{2, t-1} z_{3, t-1}\right]^{\prime}$ vector to the left side of equation (12), the VAR in (12) can be presented as follows:

$$
\left[\begin{array}{c}
z_{1, t} \\
z_{2, t} \\
z_{3, t}
\end{array}\right]-\left[\begin{array}{c}
z_{1, t-1} \\
z_{2, t-1} \\
z_{3, t-1}
\end{array}\right]+\left[\begin{array}{ccc}
0.4 & -0.8 & 0 \\
-0.1 & 0.6 & -0.8 \\
0 & -0.1 & 0.2
\end{array}\right]\left[\begin{array}{c}
z_{1, t-1} \\
z_{2, t-1} \\
z_{3, t-1}
\end{array}\right]=\left[\begin{array}{c}
\varepsilon_{1, t} \\
\varepsilon_{2, t} \\
\varepsilon_{3, t}
\end{array}\right] .
$$

Thus we have:

$$
\left[\begin{array}{c}
\Delta z_{1, t} \\
\Delta z_{2, t} \\
\Delta z_{3, t}
\end{array}\right]+\left[\begin{array}{ccc}
0.4 & -0.8 & 0 \\
-0.1 & 0.6 & -0.8 \\
0 & -0.1 & 0.2
\end{array}\right]\left[\begin{array}{c}
z_{1, t-1} \\
z_{2, t-1} \\
z_{3, t-1}
\end{array}\right]=\left[\begin{array}{c}
\varepsilon_{1, t} \\
\varepsilon_{2, t} \\
\varepsilon_{3, t}
\end{array}\right]
$$

Since the (1,3)-th and (3,1)-th elements of the VECM are zeros, we can conclude that Granger non-causality exists between $z_{1, t}$ and $z_{3, t}$. Indirect causality is also detected from $z_{1, t}$ to $z_{3, t}$ through $z_{2, t}$, due to all remaining nonzero elements. Hence causal relations indicated by the VECM are identical to these relations shown in the equivalent VAR in (12).

In addition, the rank of the impact matrix in (13) is 2, not 3. This matrix can be decomposed as follows:

$$
\left[\begin{array}{ccc}
0.4 & -0.8 & 0 \\
-0.1 & 0.6 & -0.8 \\
0 & -0.1 & 0.2
\end{array}\right]=\left[\begin{array}{cc}
-0.4 & 0 \\
0.1 & -0.4 \\
0 & 0.1
\end{array}\right]\left[\begin{array}{ccc}
-1 & 2 & 0 \\
0 & -1 & 2
\end{array}\right] \text {. }
$$

The following two ZNZ patterned cointegrating vectors are also identified:[ $\left.\begin{array}{lll}-1 & 2 & 0\end{array}\right]$ and $\left[\begin{array}{ll}0 & -1\end{array}\right]$.Further, the first selected cointegrating 
vector demonstrates that the first asset's return and the second asset's return are cointegrated. The different sign occurring in $z_{1, t-1}$ and $z_{2, t-1}$ indicates that, ceteris paribus, when the first asset's return rises, the second asset's return increases. It also implies that, ceteris paribus, a decrease in the first asset's return leads to a fall of the second asset's return. The second selected cointegrating vector indicates that the cointegrating relationship exists between the second asset's return and the third asset's return. The different sign occurring in $z_{2, t-1}$ and $z_{3, t-1}$ also reveals that, ceteris paribus, a rise in the second asset's return leads to an increase in the third asset's return. For the second example, all $z_{1, t}$, $z_{2, t}$ and $z_{3, t}$ are determined by the following equations:

$$
\begin{gathered}
z_{1, t}-z_{1, t-1}=\varepsilon_{1, t} \\
z_{2, t}+z_{1, t-1}-0.5 z_{2, t-1}-0.8 z_{3, t-1}=\varepsilon_{2, t} \\
z_{3, t}-z_{3, t-1}=\varepsilon_{3, t} .
\end{gathered}
$$

The VAR model of this system can then be shown as:

$$
\left[\begin{array}{c}
z_{1, t} \\
z_{2, t} \\
z_{3, t}
\end{array}\right]+\left[\begin{array}{ccc}
-1.0 & 0 & 0 \\
1 & -0.5 & -0.8 \\
0 & 0 & -1
\end{array}\right]\left[\begin{array}{c}
z_{1, t-1} \\
z_{2, t-1} \\
z_{3, t-1}
\end{array}\right]=\left[\begin{array}{c}
\varepsilon_{1, t} \\
\varepsilon_{2, t} \\
\varepsilon_{3, t}
\end{array}\right] .
$$

In this VAR model $a_{2,1}^{1}(L)$ and $a_{2,3}^{1}(L)$ are non-zeros, while the remaining $a_{i, j}^{1}(L), i \neq j$, are zeros. Thus Granger causality exists only from both the first asset's return and the third asset's return to the second asset's return. To determine the roots of the characteristic polynomial for inspecting unit roots, we have

$$
\operatorname{det}\left[\begin{array}{ccc}
1-L & 0 & 0 \\
L & 1-0.5 L & -0.8 L \\
0 & 0 & 1-L
\end{array}\right]=0
$$

which leads to $\operatorname{det}\left\{(1-0.5 L)(1-L)^{2}\right\}=0$. Therefore two unit roots are detected.

Next, the VAR in (14) can be presented as follows: 


$$
\left[\begin{array}{c}
z_{1, t} \\
z_{2, t} \\
z_{3, t}
\end{array}\right]-\left[\begin{array}{c}
z_{1, t-1} \\
z_{2, t-1} \\
z_{3, t-1}
\end{array}\right]+\left[\begin{array}{ccc}
0 & 0 & 0 \\
1 & 0.5 & -0.8 \\
0 & 0 & 0
\end{array}\right]\left[\begin{array}{c}
z_{1, t-1} \\
z_{2, t-1} \\
z_{, t-1}
\end{array}\right]=\left[\begin{array}{c}
\varepsilon_{1, t} \\
\varepsilon_{2, t} \\
\varepsilon_{3, t}
\end{array}\right] .
$$

Thus we have the following equivalent VECM:

$$
\left[\begin{array}{c}
\Delta z_{1, t} \\
\Delta z_{2, t} \\
\Delta z_{3, t}
\end{array}\right]+\left[\begin{array}{ccc}
0 & 0 & 0 \\
1 & 0.5 & -0.8 \\
0 & 0 & 0
\end{array}\right]\left[\begin{array}{c}
z_{1, t-1} \\
z_{2, t-1} \\
z_{3, t-1}
\end{array}\right]=\left[\begin{array}{c}
\varepsilon_{1, t} \\
\varepsilon_{2, t} \\
\varepsilon_{3, t}
\end{array}\right] .
$$

Since only (2,1)-th and (2,3)-th elements of the off-diagonal elements in the VECM are non-zeros, we can conclude that Granger causality exists only from both $z_{1, t}$ and $z_{3, t}$ to $z_{2, t}$, due to all remaining zero elements. Hence causal relations indicated by the VECM are identical to these relations shown in the equivalent VAR in (14).

Since the rank of the impact matrix in (15) is 1 , not 3 , we can have the following decomposition:

$$
\left[\begin{array}{ccc}
0 & 0 & 0 \\
1 & 0.5 & -0.8 \\
0 & 0 & 0
\end{array}\right]=\left[\begin{array}{l}
0 \\
1 \\
0
\end{array}\right]\left[\begin{array}{lll}
1 & 0.5 & -0.8
\end{array}\right] .
$$

Thus the following single $\mathrm{ZNZ}$ patterned cointegrating vector is identified:

$\left[\begin{array}{lll}1 & 0.5 & -0.8\end{array}\right]$

Further, the selected cointegrating vector demonstrates that the first asset's return, the second asset's return and the third asset's return are cointegrated. The same sign occurring in $z_{1, t-1}$ and $z_{2, t-1}$ and the different sign occurring in $z_{1, t-1}$ and $z_{3, t-1}$ indicate that, ceteris paribus, an increase in the first asset's return leads to a fall of the second asset's return, but a rise of the third asset's return.

\section{Exchange Rate Applications}

The above sections have shown ZNZ patterned VECM modeling. As argued earlier, the use of this procedure is particularly suited to 
Annual \% change

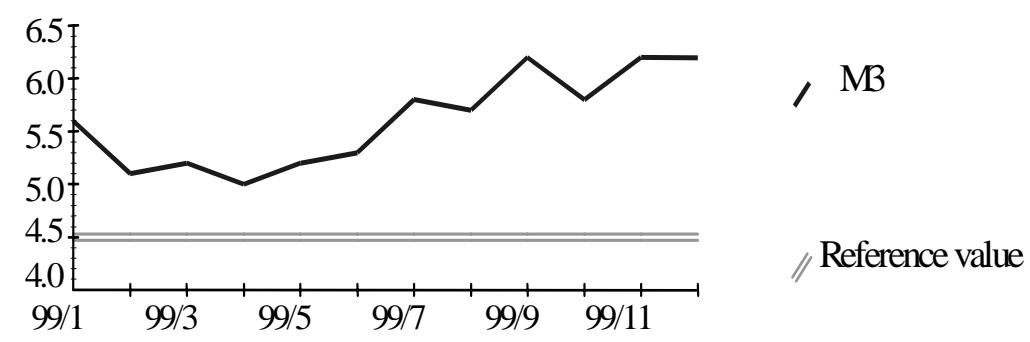

FIGURE 1.- M3 Growth and the Reference Value in the European Monetary Union Source: ECB

financial variables in which complex relationships exist. In the next two sub-sections, two empirical applications of the modeling procedure using foreign exchange examples are presented.

\section{A. Relationship between Movements of the Euro and the Money Supply}

The first application focuses on the detection of causal relationships between movements in the Euro's exchange rate (relative to the U.S. dollar) and the money supply in the European Monetary Union.

The Euro was introduced on 1 January 1999 as official currency. To date the Euro has become the second most widely traded currency at the international level, behind the U.S. dollar and ahead of the Japanese yen. During the first year of trading, the value of the Euro relative to the U.S. dollar fell markedly. The Euro's weakness throughout this period confounded earlier general expectations that it would trend upwards relative to the U.S. dollar (see ECB [2001]).

Money supply in the Euro area is measured by the standard stock of money (M3). It consists of sight deposits, shorter deposits of up to 2 years, and marketable instruments. Figure 1 shows that for the entire year of 1999 the monthly measures of M3 were always higher than the reference value of 4.5 percent set by the Governing Council of the European Central Bank. The Governing Council has adopted a price stability-oriented monetary policy strategy, that is, the rate of monetary expansion is designed to achieve the objective of price stability. Thus it may be presumed that the 4.5 percent benchmark for the growth rate of M3 is regarded as the level to accomplish this objective.

Our task is to assess the nature of the influence of the money supply 
on the exchange rate, ceteris paribus. In the gold standard era, since the gold reserves of a country were limited (if they were not gold producers), the growth rate of money supply was related to the level of country's reserves. The unmanaged growth of money supply could lead to changes in value of a country's currency. In the present circumstances of a floating exchange rate system, currencies are expected to fluctuate according to supply and demand. In order to smooth the market, governments may adjust the money supply which directly or indirectly influences the foreign exchange rate. However governments are not able to control the exchange rate over a long period without regard to economic fundamentals.

The most widely held view is that, ceteris paribus, an expansion in money supply is associated with a decrease in domestic interest rates, which leads to a depreciation in the domestic currency. Conversely, a tightening of monetary policy leads to an appreciation of the domestic currency. Lewis (1993) utilizes VAR modeling to investigate the impact of U.S. monetary shocks on the U.S. dollar exchange rate and finds that a loosening of monetary policy is associated with a depreciating currency. Cushman and Zha (1997) examine the effects of monetary shocks on the Canadian dollar, and again employ the VAR approach to conduct their tests. They conclude that a contraction in the U.S. money supply leads to a depreciation in the Canadian dollar.

There is already literature reporting the causal relationships between the money supply and economic activity in the Euro area (see BIS [2000]). However an investigation into the direct relationships between the money supply and the Euro's exchange rate has so far not been attempted. ${ }^{6}$

To investigate the causal relationships between the movements in the Euro's exchange rate (against the U.S. dollar) and the money supply of the Euro area, monthly data on the Euro's exchange rate $\left(E_{e}\right)$ and seasonally adjusted M3 are collected from DataStream ${ }^{\mathrm{TM}}$. We use data beginning at January 1997 and ending at August 2001 which provides a sample size of 56 months. ${ }^{7}$ A smaller sample size is considered to be

6. Of course, in reality the relationship between the money supply and exchange rate involves other relevant variables, including, but not limited to, international funds flows, consumer prices and interest rate differentials.

7. The Euro was in a preliminary stage prior to 1 January 1999 . The currency was an artificial construct comprising a basket - the European currency unit - that was used by the member states of the EU as their internal accounting unit for the currency area of the European Monetary System (EMS). The EMS was a managed flexible exchange rate system 
insufficient in order to conduct the analysis. In detecting the causal relationships between the movements of the Euro's exchange rate and the money supply, the optimal VECM models are selected for $\log (\mathrm{M} 3)$ and $\log \left(E_{e}\right)$ at $\mathrm{T}=56,57,58,59$ and 60 . To increase the sample size from 57 to 60 we then add a single month sequentially from September 2001 to December 2001, and re-estimate the model as we add each observation. To examine stationarity for each series, the augmented Dickey-Fuller (ADF) unit root test is used. The results show that both $\log E_{e}$ and $\log \mathrm{M} 3$ are $I(1)$ processes at $\mathrm{T}=56,57,58,59$ and $60 .{ }^{8}$

To demonstrate the usefulness of the proposed algorithms in a small sample environment, a maximum order of 12 is selected. We use a maximum lag of 12 because we expect the non-zero coefficients in the autoregressive part to have lag length less than 12, and accept the likelihood is high of a $\mathrm{ZNZ}$ patterned lag structure. We also need to keep as many degrees of freedom as possible. This bi-variate system includes coefficient matrices in the VAR part and an impact matrix. The maximum lag of 12 gives us $4^{13}=67,108,864$ possible candidate models to select the optimal VECM model.

Following the proposed algorithm, the optimal ZNZ patterned VECM models from $T=56$ to $T=60$ are chosen using the Schwarz Bayesian Criterion (SBC). ${ }^{9}$ The optimal models selected are estimated using the GLS techniques and are shown in table $1 .{ }^{10}$ To check the adequacy of each optimal model fit, the strategy suggested in Tiao and Tsay (1989) and Penm et al. (1997) is used, with the proposed Penm and Terrell (1984b) algorithm applied to test each residual vector series, using the SBC criterion. ${ }^{11}$ The results in table 1 support the hypothesis

that defined bands wherein the bilateral exchange rates of the member countries could fluctuate. In this sense the data pre-1999 are not true market-determined rates but rather indicative figures.

8. To preserve journal space, the relevant test results are not presented here but can be supplied on request.

9. The zero entries are determined from the data using the model selection criteria to determine the optimal ZNZ patterned VECM model. Details are provided in the appendices.

10. In a simultaneous equation system GLS estimates are more efficient than OLS estimators, when the regressors in each individual equation are not identical (Zellner [1962]). Since the VECM is a simultaneous equation system, and the ZNZ pattern is extremely unlikely to produce the same regressors in each equation, GLS techniques will be necessary in most cases.

11. In ZNZ patterned time-series modeling Tiao and Tsay (1989) propose an algorithm 
that each residual vector is a white noise process. These optimal models are then used as the benchmark models for analyzing the causal relationships. In addition, in conducting the instantaneous causality detection, the algorithm proposed in Penm and Terrell (1984b) is also applied to the estimated $V$ of each optimal model. ${ }^{12} 13$

In analyzing the causality detected, a patterned VECM which shows Granger-causality from $\mathrm{M} 3$ to $E_{e}$, Granger no-causality from $E_{e}$ to $\mathrm{M} 3$, and no instantaneous causality between M3 and $E_{e}$ is selected at all times. This outcome confirms that the money supply influences the movements of the Euro over the test period. M3 is detected as a variable, which produces leading information on the Euro's movements. That is, a shock to M3 creates a lagged response in the Euro. These findings are consistent with economic intuition and prior evidence.

\section{B. Purchasing Power Parity in Japan}

The second application examines purchasing power parity (PPP) using the bilateral exchange rates between the Japanese yen and the U.S. dollar. The PPP theory states that movements in the exchange rate between two countries' currencies are determined by movements in their relative prices. Formally the PPP condition can be expressed as $E_{t}$ $=P_{t} / P_{t}^{*}$, where $E_{t}$ denotes units of domestic currency per unit of foreign currency, $P_{t}$ domestic price level, and $P_{t}^{*}$ foreign price level.

Recently, cointegration has been widely utilized to test for PPP. Following Engle and Granger (1987), consider an I(1) system where both $\log \left(E_{t}\right)$ and $\log \left(P_{t} / P_{t}^{*}\right)$ are characterized as integrated of order 1. If there is a long-term cointegrating relationship between them, that is $\beta^{\prime} X_{t}=\left(\beta_{1}, \beta_{2}\right)\left[\log \left(E_{t}\right), \log \left(P_{t} / P_{t}^{*}\right)\right]^{\prime}=\mathcal{E}_{t}$ with $\mathcal{E}_{t}$ as a stationary process,

using the $\operatorname{crit}(m, j)$ criterion to select the vector autoregressive moving average process with zero entries. After the final model is selected, their algorithm is then applied to the residual series to test whether this series is a vector white noise process.

12. It is useful to re-estimate the model parameters using the sample sizes of $\mathrm{T}=56,57$, 58, 59 and 60. Since we achieved a similar specification for all sample sizes under examination. This supports a constant parameter specification with identical ZNZ patterns showing that our specification results are not changing as we include additional observations.

13. Instantaneous causality indicates the interactions among contemporaneous variables involved in the system (see Chow [1983]). 
TABLE 1. The VECM's ${ }^{a, b}$ Selected for Detecting the Causal Relationships between $E_{e}$ and $M 3$

VECM: $\Delta y(t)+\sum_{\tau-1}^{p-1} A_{\tau}^{*} \Delta y(t-\tau)+A^{*} y(t-1)=\varepsilon(t)$,

where $y(t)=\left[\log E_{e}, \log \mathrm{M} 3\right]^{\prime}$.

\begin{tabular}{llll}
\hline Sample size $(\mathrm{T})$ & 56 & 57 & 58 \\
\hline Value of $\tau$ selected & 1 & 1 & 1
\end{tabular}

Estimated $A_{1}^{*}$

$\left[\begin{array}{cc}-0.3801 & 0 \\ (0.1246) & \\ 0 & 0\end{array}\right] \quad\left[\begin{array}{cc}-0.3728 & 0 \\ (0.1229) & \\ 0 & 0\end{array}\right] \quad\left[\begin{array}{cc}-0.3609 & 0 \\ (0.1233) & \\ 0 & 0\end{array}\right]$

Estimated $A^{*}$
$\left[\begin{array}{cc}0.2006 & 0.0299 \\ (0.0636) & \\ 0 & 0\end{array}\right]\left[\begin{array}{cc}0.2009 & 0.0316 \\ (0.0631) & \\ 0 & 0\end{array}\right]\left[\begin{array}{cc}0.1981 & 0.0288 \\ (0.0640) & \\ 0 & 0\end{array}\right]$

Value of $p$

for residual

analysis: (Normalized $\mathrm{SBC}^{\mathrm{c}}$ )

57

$\begin{array}{llll}0 & 1 & 2 & 3\end{array}$

1. $1.007 \quad 1.025 \quad 1.034$

58

$\begin{array}{llll}0 & 1 & 2 & 3\end{array}$

1. $1.006 \quad 1.017 \quad 1.025$

59

$\begin{array}{llll}0 & 1 & 2 & 3\end{array}$

1. $1.007 \quad 1.018 \quad 1.025$

Pattern of Granger

causality $^{\mathrm{d}} \quad \log E_{e} \leftarrow \log \mathrm{M} 3 \quad \log E_{e} \leftarrow \log$ M3 $\quad \log E_{e} \leftarrow \log$ M3 
TABLE 1. (Continued)

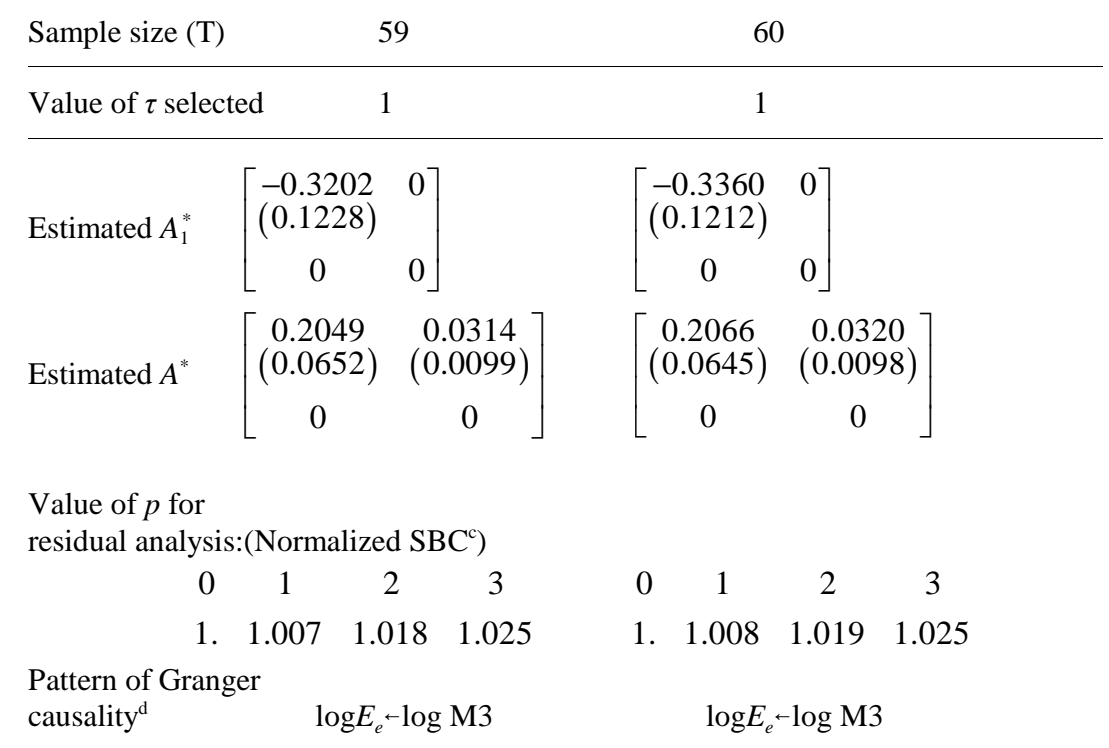

Note: The VECM model is a: The value of $p$ is chosen by AIC, in conjunction with confirming the residual vector is a white noise process; $b$ : Model selected by SBC using the GLS Procedure. Standard errors are in parentheses. $\Delta$ denotes first difference. c: For simplicity, the values of SBC for $p>3$ are not presented, but can be supplied to readers upon request. d: $w \rightarrow z$ denotes $w$ Granger causes $z$.

then PPP holds in the long run. ${ }^{14}$

In contrast to the commonly employed unit-root based tests and fullorder VECM modeling, the ZNZ VECM modeling presents a single framework for conducting causality detection and cointegration investigation among the variables involved in the system, and provides estimates of the long-term and dynamic responses.

Prior research has shown that high-frequency data (for example monthly data) may not reveal evidence of PPP in the long run (see McNown and Wallace [1989], Taylor [1988], and Corbae and Ouliaris [1998]). However when researchers (see Edison [1987], and Kim [1990]) shift to low-frequency data such as an annual series, and use

14. For this case, McFarland et al. (1994) claim that the necessary condition for PPP holds in the long run. If the cointegrating vector is $\beta^{\prime}=(1,-1)$, the necessary and sufficient condition for PPP will hold. 


\section{TABLE 2. The VECM ${ }^{\mathrm{a}, \mathrm{b}}$ Identified for Examining PPP in Japan}

Variables: $y_{1}(t)=\log (E), y_{2}(t)=\log (P), y_{3}=\log (I R)$.

Sample Period: 1974 to 2000;

VECM: $A^{*} y(t-1)+\Delta y(t)+\sum_{\tau=1}^{p-1} A_{\tau}^{*} \Delta y(t-\tau)=\varepsilon(t)$.

Non-zero $(i, j)$-th entries in estimated coefficient matrices, $A_{\tau}^{*}$ and $A^{*}$ :

$\begin{array}{llrlclc}\tau & i, j & \text { entry (s.e.) } & i, j & \text { entry (s.e.) } & i, j & \text { entry (s.e.) } \\ 1 & 2,3 & 0.1267(0.0584) & 3,3 & -0.1258(0.0531) & & \\ 2 & 2,2 & 0.1732(0.0497) & 2,3 & 0.1197(0.0562) & & \\ 5 & 2,3 & -0.1093(0.0583) & 3,1 & 0.1896(0.0553) & & \\ 6 & 2,3 & -0.1463(0.0581) & & & & \\ 7 & 3,1 & 0.1252(0.0550) & & & & \\ 10 & 2,2 & 0.1504(0.0502) & 2,3 & 0.1106(0.0561) & & \\ 11 & 1,1 & -0.1179(0.0558) & & & & \\ 12 & 2,2 & -0.3058(0.0513) & & & & \\ 13 & 2,3 & -0.1835(0.0561) & & & & \\ 16 & 1,3 & -0.1168(0.0532) & 3,3 & 0.1026(0.0530) & & \\ A^{*} & 2,1 & -0.0545(0.0121) & 2,2 & 0.0178(0.0040) & 2,3 & 0.0647(0.0128)\end{array}$

The type of $\hat{\mathrm{V}}$ selected: $\left[\begin{array}{ccc}1.10103 E-03 & 0 & 0 \\ 0 & 2.81048 E-05 & 0 \\ 0 & 0 & 1.13040 E-05\end{array}\right]$

Residual analysis

$\begin{array}{lllllll}\text { Existing lags } & 0 & 1 & 2 & 3 & 4 & 5\end{array}$

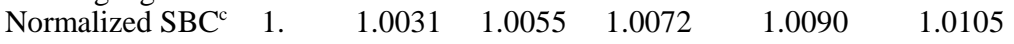

Long-term

Cointegrating Relationship Identified: $\log (E)=0.3281 \log (P)+1.1853 \log (I R)$

Note: a)The value of $p$ is chosen by AIC, in conjunction with confirming the residual vector is a white noise process. b) Model selected by SBC using the GLS procedure. Standard errors in parentheses. $\Delta$ denotes first difference. c) For simplicity, the values of SBC for $p>5$ are not presented, but can be supplied to readers upon request. Of note, the SBC criterion is the sum of two terms. The first term is the log of the determinant of the estimated residual variance-covariance matrix, while the second term depends on the number of functionally independent parameters estimated and a term which is $\log$ (sample size)/sample size. In this paper all SBC values computed for the two applications are positive values. No negative values have been obtained. To conserve space and to avoid cluttering we use the normalized SBC values. Since all SBC values are positive in this paper, the smallest normalized SBC is used to determine the lag parameter $p$.

cointegration techniques to test the PPP, empirical evidence usually supports the long run PPP hypothesis. ${ }^{15}$

15. Examples of using cointegration techniques to find evidence of PPP include Fleissig 
For the test, monthly averaged data over the period 1974/1 to 2000/12 for the following three variables are obtained from DataStream $^{\mathrm{TM}}$ :

Japanese yen to U.S. dollar: exchange rate $(E)$ per U.S. dollar, Japanese CPI to U.S. CPI: ratio of price levels $(P)$,

$(1+\mathrm{U} . \mathrm{S}$. discount rate $) /(1+\mathrm{Japanese}$ discount rate): interest rate ratio $(I R){ }^{16}$

The $y$ vector comprises $\log (E), \log (P)$, as well as $\log (I R)$. The unit root tests indicate that all three variables are $I(1) .{ }^{17}$ To select the optimal ZNZ patterned VECM, we start with a maximum lag of 24 to search the optimal subset VECM model. Among the $2^{25}$ possible candidate subset VECM models, the optimal subset VECM selected has a maximum lag of 16. As we use monthly observations VECM modeling is able to accommodate both long-term and dynamic responses including seasonal effects. Thus it is reasonable that the search algorithm has produced a maximum lag of 16.

Given the framework of this subset VECM, we then select the optimal ZNZ patterned VECM in terms of model selection criteria. ${ }^{18}$ The optimal patterned VECM identified is presented in table 2.

The presence of the long-term cointegrating relationships shown in table 2 is consistent with PPP holding within the $I(1)$ system and across the Japanese and U.S. exchange markets. ${ }^{19}$ The selected pattern of the cointegrating vector also demonstrates some interesting findings. In relation to PPP, the positive relation between $\log (E)$ and $\log (P)$ and the positive relation between $\log (E)$ and $\log (I R)$ indicate that an increase in $P$ or an increase in $I R$ leads to a depreciation of the yen. This result is

and Strauss (2000), Taylor and Sarno (1998), Papell (1997), Lothian (1997), and Oh (1996).

16. Consistent with the Fisher equation, the interest rate ratio is expressed in this form and is numerically less variable than the simple ratio of percentage rates. Recently Cheng (1999) has included the interest ratio variable when he conducts PPP testing and causality detection. Cheng's analysis concerns PPP between the U.S.A. and Japan using annual data, and he finds evidence supporting PPP in the long run. In order to compare our findings with Cheng's results, the interest ratio variable has been included in the analysis.

17. Since the interest rate ratio has characteristics consistent with an evolutionary process, it is reasonable that this series is $I(1)$. The unit root tests show that the variable, $(1+$ U.S. discount rate), is $I$ (1) and so is the variable, (1+Japanese discount rate). In this instance we also find that the ratio of these two variables is $I(1)$.

18. These model selection criteria are the combination of a measure for in-sample fitting and a scaled penalty for over use of parameters (see Hannan and Deistler [1988]). Thus in-sample fitting results have played a part in the model selection criteria decision making.

19. Cheng's analysis also finds evidence of cointegration among these series. 
Frequency

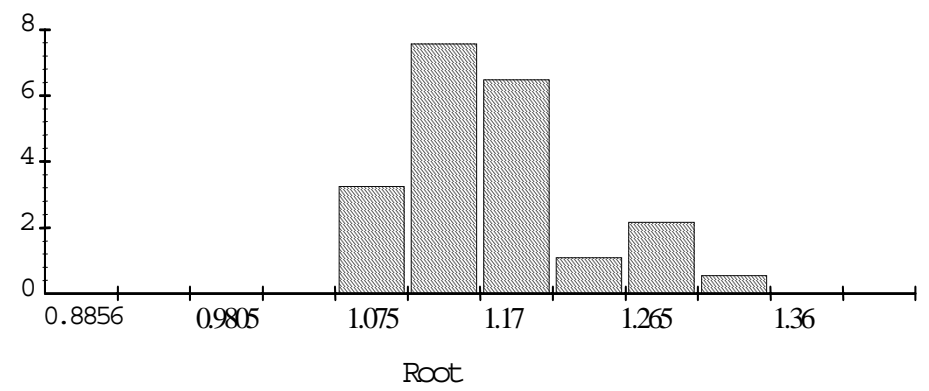

FIGURE 2. - Histogram of Roots for the VECM Shown in Table 2 Minimum: 1.052 Median: 1.143 Maximum: 1.336

consistent with economic intuition. For instance, when the price level in Japan is higher relative to the price level in the U.S., the yen would depreciate in order to retain PPP. Further, when the interest rate in Japan relative to the interest rate in the U.S. decreases, there is an associated depreciation of the yen.

In reference to the Granger causal relations among the variables, feedback relations exist between the pair of $\log (P)$ and $\log (I R)$, and the pair of $\log (E)$ and $\log (I R)$. Direct Granger causation exists from $\log (E)$ to $\log (P)$. Although there is no direct Granger causation from $\log (P)$ to $\log (E)$, indirect causation exists from $\log (P)$ to $\log (E)$ via $\log (I R)$. We therefore conclude that a Granger causal relation (directly or indirectly) exists between $\log (E)$ and $\log (P)$. Hence, the feedback within the system is complete and shocks to any one of the variables will be transmitted through the system. In addition, no instantaneous causality is detected among the variables.

Figure 2 shows that all roots detected lie outside the unit circle $(>1)$. This latter finding shows that the selected model has no unit roots or unstable roots. Thus the model is a stable one. Also a strong dynamic structure is found, and cointegrating vectors are identified. Complete causality patterns, including both non-causality and indirect causality are also given. Therefore the model is a powerful one. Also, to check the adequacy of the model fit, the results in table 2 support the hypothesis that the residual vector is a white noise process.

\section{Conclusion}

In this paper three contributions have been made in the analysis of vector financial time series where causality detection and cointegration 
investigation are important. First, we have shown that $\mathrm{ZNZ}$ patterned VECM modeling not only accommodates long-term and dynamic responses for analyzing cointegrating relations, but also provides a single framework for detecting direct and indirect causality among the variables. Compared with full-order VECM modeling, patterned VECM modeling is a more effective means of causality detection and the associated cointegration investigation for time series of integrated order $I(d)$, where the structure is truly patterned.

Second, the paper shows how, in a limited context, ZNZ patterned VECM modeling can be applied to studying the relationships among financial variables. Specifically, the evidence here shows that money supply (M3) is a source of financial and economic influence on the Euro.

Third, a general outcome of previous studies indicates that long-term PPP may not hold with high-frequency data. In this paper, support for PPP is found using monthly data between Japan and the U.S. The findings indicate that both direct and indirect causality exist among prices, interest rates and the exchange rate. This evidence sheds light on the adjustment mechanisms through which PPP is achieved. In addition, it is clear that the proposed ZNZ patterned VECM modeling allows better modeling insights in conducting financial time-series analysis.

\section{References}

Ahn, S.K., and Reinsel, G.C. 1990. Estimation for partially nonstationary multivariate autoregressive model. Journal of the American Statistical Association 85: 815-823.

Bank for International Settlements 2000. 70th Annual Report (March).

Barnhill Jr., T.M.; Joutz, F.L. and Maxwell, W.F. 2000. Factors affecting the yields on non-investment grade bond indices: A cointegration analysis. Journal of Empirical Finance 7: 57-86

Chen, S-L. and Wu, J-L. 2000. A re-examination of purchasing power parity in Japan and Taiwan. Journal of Macroeconomics 22: 271-284.

Cheng, B.S. 1999. Beyond the purchasing power parity: Testing for cointegration and causality between exchange rates, prices, and interest rates. Journal of International Money and Finance 18: 911-924

Chow, G.C. 1983. Econometrics. New York: McGraw-Hill.

Christoffersen, P.F. and Diebold, F.X. 1998. Cointegration and long horizon forecasting. Journal of Business and Economic Statistics 16: 450458.

Corbae, D. and Ouliaris, S. 1988. Cointegration and tests of purchasing power parity. Review of Economics and Statistics 70: 508-511.

Cushman, D.O. and Zha, T. 1997. Identifying monetary policy in a small open economy under flexible exchange rates. Journal of Monetary Economics 39: 433-448. 
Edison, H.J. 1987. Purchasing power parity in the long-term: A test of the dollar/pound exchange rate (1890-1978). Journal of Money, Credit and Banking 19: 376-387.

Engle, R.F. and Granger, C.W.J. 1987. Cointegration and error-correction, representation, estimation and testing. Econometrica 55: 69-104.

Engle, R.F. and Yoo, B.S. 1987. Forecasting and testing in co-integrated system. Journal of Econometrics 35: 143-159.

European Central Bank 2001. Monthly Bulletin (February).

Fleissig, A.R. and Strauss, J. 2000. Panel unit root tests of purchasing power parity for price indices. Journal of International Money and Finance 19: 489-506.

Granger, C.W.J. 1981. Some properties of time series data and their use in econometric model specification. Journal of Econometrics 16: 121-130.

Hannan, E.J. and Deistler, M. 1988. The Statistical Theory Of Linear Systems. New York: John Wiley and Sons.

Harris, R. 1995. Using Cointegration Analysis in Econometric Modeling. Prentice Hall.

Hsiao, C. 1982. Autoregressive modeling and causal ordering of economic variables. Journal of Economics Dynamics and Control 4: 243-259.

Johansen, S. 1988. Statistical analysis of cointegration vectors. Journal of Economic Dynamics and Control 12: 231-255.

Johansen, S. 1991. Estimation and hypothesis testing of cointegrating vector in gaussian vector autoregression models. Econometrica 59: 1551-1580.

Johansen, S. 1992. Cointegration in partial systems and the efficiency of single equations analysis. Journal of Econometrics 52: 389-402.

Johansen, S. 1995. A statistical analysis of cointegration for I(2) variables. Econometric Theory 11: 25-29.

Kim, Y. 1990. Purchasing power parity in the long run: a cointegration approach. Journal of Money, Credit and Banking 22: 491-503.

King, R.; Plosser, C.; Stock, J.H. and Watson, M.W. 1991. Stochastic trends and economic fluctuations. American Economic Review 81: 819-840.

Lee, B.S. 1996. Comovements of earnings, dividends, and stock prices. Journal of Empirical Finance 3: 327-346.

LeSage, J.P. 1990. A comparison of the forecasting ability of ECM and VAR models. Review of Economics and Statistics 72: 664-71.

Lewis, K.K. 1993. Are foreign exchange intervention and monetary policy related and does it really matter? No. 4377, NBER Working paper.

Lothian, J.R. 1997. Multi-country evidence on the behavior of purchasing power parity. Journal of International Money and Finance 16: 19-35.

MacDonald, R. and Power, D. 1995. Stock prices, dividends and retention: Long-term relationships and short-term dynamics. Journal of Empirical Finance 2: 135-151.

McFarland, J.W.; McMahon, P.C. and Ngama, Y. 1994. Forward exchange rates and expectations during the 1920's: A re-examination of the evidence. Journal of International Money and Finance 13: 627-36.

McNown, R. and Wallace, M.S. 1989. National price levels, purchasing power parity, and cointegration: A test for four high inflation economies. Journal 
of International Money and Finance 8: 533-545.

Oh, K.Y. 1996. Purchasing power parity and unit root tests using panel data. Journal of International Money and Finance 15: 405-18

Papell, D. 1997. Searching for stationarity: Purchasing power parity under the recent float. Journal of International Economics 43: 313-332.

Paulsen, J. 1984. Order determination of multivariate autoregressive time series with unit root. Journal of Time Series Analysis 5: 115-127.

Penm, J.H.W. and Terrell, R.D. 1984a. Multivariate subset autoregression. Communications in Statistics 13: 449-61.

Penm, J.H.W. and Terrell, R.D. 1984b. Multivariate subset autoregressive modeling with zero constraints for detecting causality. Journal of Econometrics 3: 311-30.

Penm, J.H.W. and Terrell, R.D. 1997. The selection of ZNZ patterned cointegrating vectors in error-correction modeling. Econometric Reviews 16: 281-304.

Pötscher, B.M. 1989. Model selection under nonstationarity: Autoregressive model and stochastic linear regression models. The Annals of Statistics 7: 12571274.

Reinsel, G.C. and Ahn, S.K. 1992. Vector autoregressive models with unit roots and reduced rank structure: Estimation, likelihood ratio test, and forecasting. Journal of Time Series Analysis 13: 353-375.

Schwarz, G. 1978. Estimating the dimension of a model. The Annals of Statistics 6: 461464.

Stock, J.H. and Watson, M.W. 1993. A simple estimator of cointegrating vectors in higher order integrated systems. Econometrica 61: 783-820.

Taylor, M.P. 1988. An empirical examination of long-term purchasing power parity using cointegration techniques. Applied Economics 20: 1369-1381.

Taylor, M.P. and Sarno, L. 1998. Real exchange rates under the recent float: Unequivocal evidence of mean reversion. Economics Letters 60: 131-137.

Tiao, G.C. and Tsay, R.S. 1989. Model specification in multivariate time-series. Journal of the Royal Statistical Society B 51: 157-213.

Zellner, A. 1962. An efficient method of estimating seemingly unrelated regressions and tests for aggregation bias. Journal of the American Statistical Association 57: 348-368. 